\title{
Use of mechanical resonance in impact machines
}

\author{
Wieslaw Fiebig ${ }^{1, *}$, Jakub Wrobel ${ }^{1, *}$ \\ ${ }^{1}$ Wroclaw University of Science and Technology, Department of Mechanical Engineering, 50-370 \\ Wroclaw, Lukasiewicza 7/9, Poland
}

\begin{abstract}
An innovative method exploiting mechanical resonance in machines drive systems, especially useful in impact machines, has been developed. Accumulation of energy at resonance can be applied to the drive system in a similar way as flywheels in eccentric presses. Under resonance conditions, the total energy consumption of the oscillating mass is equal to the energy lost due the damping forces. Energy accumulated in the oscillator can be several times greater than the energy supplied continuously to the oscillator. The developed method can be used in many applications, especially in impacting machines. Finally, the energy demand of resonance punching press will be compared with the energy demand of eccentric press.
\end{abstract}

\section{Introduction}

The vibration amplitude of a mechanical oscillating system depends on the ratio of the driving force frequency and the natural frequency of the system. When the excitation force frequency is equal to the natural frequency of the object, the phenomenon of mechanical resonance occurs, and as a result strong increases in the vibration amplitude can be observed [1-4]. The maximum vibration amplitudes depend on the damping occurring in the system.

Resonance in mechanical systems is in most cases unwanted. Increased vibrations lead to higher dynamic loads acting on the components of the mechanical systems. Overload and fatigue may strongly influence the integrity of a construction or structure e.g. the Tacoma Bridge, footbridges, shaft critical speeds, etc. In contrast to mechanical systems, the positive effects of resonance are used in many other applications such as acoustics, medicine (magnetic resonance), electrical machines, radio, laser technology, etc. [1].

Resonance is used in a new drilling technique [2] called resonance hammer drilling (RHD) as an alternative to increase the rate of penetration in hard rocks drilling. This technique use the already existent axial vibration due to the cutting process, to generate a harmonic load on the bit and an excitation in a steel mass (hammer). When this excitation frequency is close to the steel mass natural frequency impacts on the bit occur.

Mechanical resonance is used in vibratory conveyors [3]. The experimental results show that the resonant mode of vibration conveyor with electromagnetic excitation is very advantageous, since then it consumes the least energy to maintain the system in a state of oscillation.

\footnotetext{
*Corresponding author: wieslaw.fiebig@pwr.edu.pl
} 
The paper [4] presents the case of a robotic arm where, due to the use of resonance, energy savings up to $56 \%$ were achieved. Pick-and-place robots, for example, should accelerate and decelerate not by means of large, strong actuators, but by an exchange between kinetic and potential energy. The positive effects resulting from mechanical resonance are also used in the development of the optimal parameters of micro-propulsion systems to drive flying objects [5]. Resonant excitation can offer in such systems a increased lift output, with less battery power.

The energy flow at resonance conditions has been investigated in [12-14] and it has been stated that the absorbed average mechanical power evolution can be used to describe the dynamical behavior of the vibratory system.

In this paper the use of mechanical resonance in impact machines, such as punching or stamping presses, riveting machines etc. will be described. The accumulation of the energy in the flywheel and in the oscillator at resonance will be explained. A prototype of a resonance punching machine was built and its power demand has been compared with the power demand of the conventional eccentric press.

\section{Background}

The maximum vibration amplitude of the harmonic oscillator can be determined from the formula [7]:

$$
A_{\max }=\frac{\alpha_{0}}{2 \beta \omega_{d}}=\frac{F_{0}}{2 m \beta \omega_{r}}
$$

where $\beta=\frac{c}{2 m}$ - damping coefficient, $\omega_{r}=\sqrt{\frac{k}{m}-\beta^{2}}$ - resonance frequency, $m$ - mass, $c$ damping coefficient, $F_{0}$ - amplitude of excitation force.

The increase in amplitude is accompanied by an increase in the energy of the oscillator, which can be determined from the formula:

$$
E_{a c r}=\frac{1}{2} k A_{\max }^{2}=\frac{1}{2} m \omega_{r}^{2} A_{\max }^{2}
$$

The mechanical energy will be accumulated at resonance. In the steady state conditions of resonance, the excitation force is used to overcome the damping force only. The inertia force is compensated by the force in the spring [9]. During sequential reception of energy from the oscillator at resonance, instantaneous driving force, typically of relatively low amplitude, is converted to high amplitude power pulses.

The energy supplied to the oscillator in the mechanical resonance state is several times smaller than the energy accumulated in this oscillator and is equal to the energy dissipated due to the damping [9]. In the case of a mechanism with a flywheel, the energy supplied to the system is equal to the kinetic energy of the wheel.

$$
E_{k f l}=\frac{1}{2} I \omega^{2}=\frac{1}{2} m \omega^{2} r^{2}
$$

where: $m$ - mass of the flywheel, $r$-radius of the flywheel, $\omega$ - angular velocity.

If Eq. (2) and (3) are compared, one can state that the energy of the oscillator and the kinetic energy of the flywheel are equal, when the masses and frequencies (oscillator, flywheel), are equal, and the flywheel radius corresponds to the oscillator amplitude. 


\section{Resonance press vs. eccentric press}

In Figure 3 the schematic view of the eccentric press and resonance press has been shown. In the eccentric press the energy needed in the punching process is stored in the flywheel. By activating the clutch, the press slider is displaced, and the punching operation is carried out. The main element of the resonance press is the resonant block moving on linear guides and attached to the housing by means of springs. The frequency of the driving force should be adjusted to the natural frequency of the resonance block. The driving element is an electric motor with a rotating mass that excites vibrations of the resonance block or a pneumatic or electric actuator. The natural frequency of the resonance press can be changed by changing the stiffness of the springs or changing the mass of the resonance block. The maximum punching force depends on the mass of the resonance block and its maximum vibration amplitude.

During the punching operation some energy will be extracted from the resonance block and then its amplitude is rebuilt. The vibration parameters of the resonance block can be selected so that the reconstruction of the maximum vibration amplitude occurs in every cycle or after 1 or 2 vibration cycles. The time needed to rebuild the energy in the resonance block to the maximal value is dependent mainly from the friction/damping and the amplitude of the excitation force. The vibration amplitude of the resonance block should not decrease more than $10 \%$, similar as the speed fluctuation ratio of flywheels in eccentric presses.

a)

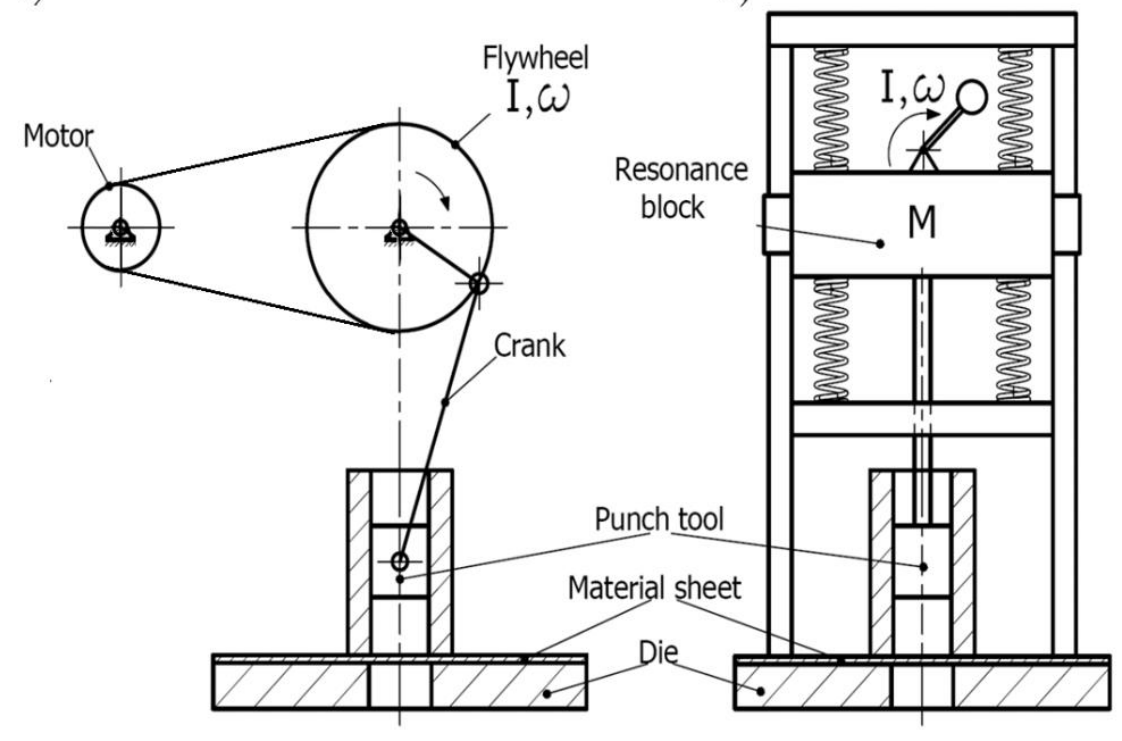

Figure 3. Schematic view of: a) eccentric press b) resonance press.

The energy accumulation at resonance can be used therefore in drive systems of impact working machines in similar way as flywheels. 


\subsection{Prototype of resonance press}

Figure 4 presents the schematic 3D model and view of a prototype of mechanical press. The resonance block (4) is excited with the resonance frequency with use of rotational mass (9). The electric motor (1) is connected to a gearbox (5) where the rotational velocity is reduced. The work sequence at the resonance is activated at the point of maximum kinetic energy. The oscillator mass is connected to the punch element via a controlled clutch (8), which is only engaged during the punching action.

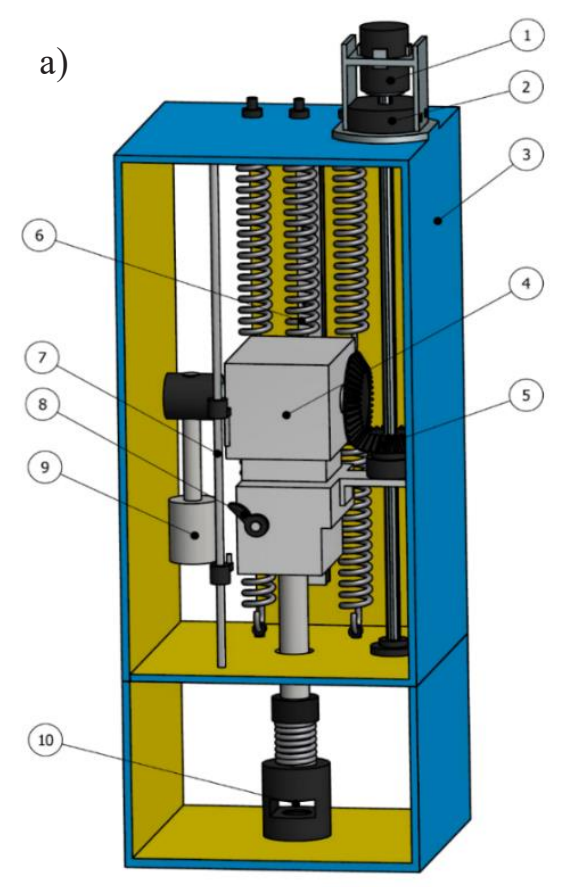

b)

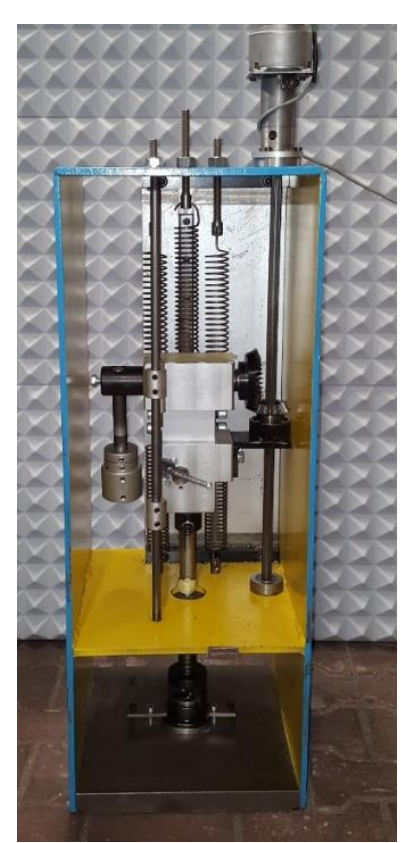

Figure 4. Prototype of the resonance punching machine a) 3D model: 1-electric motor, 2-gearbox, 3housing, 4 - oscillator mass, 5-bevel gears, 6- set of springs, 7- punch clutch triggers, 8 - clutch engage switch, 9- rotational mass, 10- punching device, b) photo.

Parameters of resonance press are the following (Table 1):

Table 1. Parameters of resonance press

\begin{tabular}{c}
\hline Resonance press \\
\hline Operating frequency: $\mathrm{f}=2.9 \mathrm{~Hz}$ \\
Mass of the resonant block: $\mathrm{m}=12 \mathrm{~kg}$ \\
Rpm of the rotating mass: $\mathrm{n}=174 \mathrm{rpm}$ \\
\hline
\end{tabular}

The mechanical clutch 8 is switched on after reaching the maximal amplitude of vibrations of the resonance block 4 . The mass of resonance block 4 is a set of all masses in the oscillating motion, i.e. the mass of the resonance block, rotating mass and tool mass. It is possible to electronically control the clutch enclosing the punching tool using a sensor to measure the displacement of the resonance block. 


\subsection{Eccentric press}

In Figure 5 the investigated eccentric press has been shown. The flywheel is driven with the electric motor and the belt drive. The flywheel is connected to the eccentric shaft, rotating in a connecting rod. The connecting rod moves a ram in a slider joint vertically.

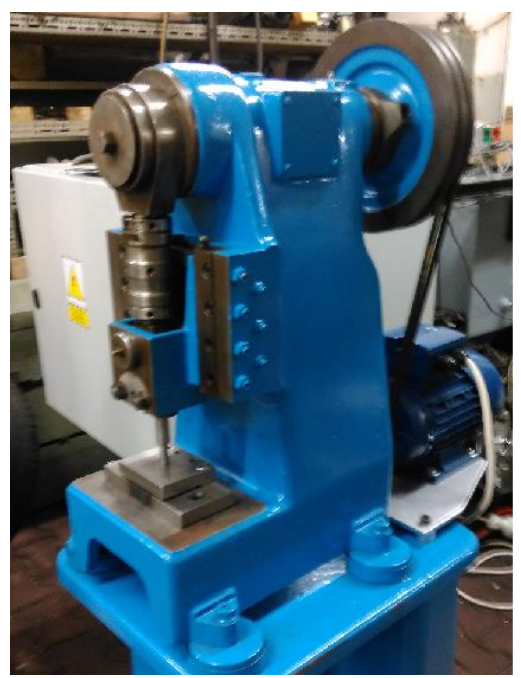

Figure 5. View of investigated eccentric press

The parameters of the eccentric press are following (Table 2):

Table 2. Parameters of the eccentric press

\section{Eccentric press}

Operating frequency: $\mathrm{f}=2.8 \mathrm{~Hz}$

Flywheel rpm: $\mathrm{n}=177 \mathrm{rev} / \mathrm{min}$

Engine rpm $\mathrm{n}=886 \mathrm{rpm}$

Flywheel mass: $\mathrm{m}=18 \mathrm{~kg}$

During the punching process, the thickness of the sheet and the diameter of the punched holes as well as the course of the punching process were the same for both presses. The sheet was continuously displaced in the die in a manner that ensures cyclical hole performance.

\subsection{Comparison of energy and power demand}

The parameters of both presses are selected in such a way that the energy supplied by the flywheel in the eccentric press is close to the energy which is accumulated in the resonance block of the eccentric press. Measurement of DC motor power in the case of a resonance press was determined based on the average current and voltage values present on motor:

$$
P=U_{r m s} \cdot I_{r m s}
$$

where $U_{r m s}$ - effective voltage value on the motor, $I_{r m s}$ - the effective value of the current flowing in the motor windings.

The power demand of the three-phase motor driving the eccentric press was measured by means of a wattmeter. The results are presented in Table 3: 
Table 3. Results of power measurements

\begin{tabular}{lc}
\hline Press type & Power demand $[\mathrm{W}]$ \\
\hline Resonance press & 59 \\
Eccentric press & 169 \\
\hline
\end{tabular}

From Table 3 is to see that the power demand of resonance press is around 2,9 times lower than the power demand of eccentric press.

\section{Conclusions}

This paper presents a new concept for the drive of impact machines with the use of mechanical resonance. It uses the effect of energy storage during resonance which is similar to energy storage in flywheels. The efficiency of a resonance press depends mainly on the damping of vibration of the resonance block. The smaller the damping, the greater the efficiency of work. The measurements show that the power demand of the resonance press is about 2,9 times lower than for the eccentric press when the same punching operation is performed. This is due to the fact that in the resonance press the inertia forces are compensated by the forces in spring elements. In the resonant press there are fewer friction resistances than in the eccentric press. In the resonance press there is no need to change the rotary motion into a reciprocating motion. The big advantage of the resonance press is the simplicity of design. The concept described in this paper can be used in many other impact machines.

\section{References}

[1] M.J. Crocker, Handbook of Noise and Vibration Control, John Wiley \& Sons, Hoboken, New Jersey, 528-545, (2007)

[2] R. Aguiar, H.I. Weber, Development of vibroimpact device for the resonance hammer drilling, Proc. Of the XII Int. Symposium of Dynamic Problems of Mechanics (DINAME) Feb. 26 - March 02, (2007)

[3] Z. Despotovic, A. Ribic, A comparison of Energy Efficiency of SCR Phase Control and Switch Mode Regulated Vibratory Conveying Drives. IX Symposium Industrial Electronics, INDEL, 2012, Banja Luka, Nov. 01-03, (2012)

[4] M.C. Plooij, M. Wisse, A Novel Spring Mechanism to Reduce Energy Consumption of Robotic Arms, Intelligent Robots and Systems (IROS), IEEE/RSJ International Conference, 2901- 2908, (2012)

[5] S. Baek, K. Ma, R. Fearing: Efficient Drive of Flapping- Wing Robots. IEEE/RSJ International Conference on Intelligent Robots and Systems, St. Louis, USA, (2009)

[6] P. Glynne-Jones, M.J. Tudor, S.P. Beeby, N.M. White, An electromagnetic, vibrationpowered generator for intelligent sensor systems, Sensors and Actuators, A 110, 344 $349,(2014)$

[7] W. Fiebig, J. Wrobel, Simulation of Energy Flow at Mechanical Resonance. 22nd ICSV Conference, July 12-16, Florence, Italy, (2015)

[8] M. Horodinca, N.E. Saghedin, Experimental Investigations of Power Absorbed at Mechanical Resonance, Experimental Techniques SEM, 1-11, (2011).

[9] W. Fiebig, J. Wrobel, Use of mechanical resonance in machines drive systems. $24^{\text {th }}$ ICSV Conference, July 23-27, London, UK., (2017) 